\title{
User Control in Adaptive User Interfaces for Accessibility
}

\author{
Matthias Peissner ${ }^{1}$ and Rob Edlin-White ${ }^{2}$ \\ ${ }^{1}$ Fraunhofer Institute for Industrial Engineering IAO, Nobelstr. 12, 70569 Stuttgart, Germany \\ ${ }^{2}$ Human Factors Research Group, ITRC Building, The University of Nottingham, \\ University Park, Nottingham, NG7 2RD, UK \\ matthias.peissner@iao.fraunhofer.de, epxrwe@nottingham.ac.uk
}

\begin{abstract}
Adaptive user interfaces offer great potential for improving the accessibility of interactive systems. At the same time, adaptations can cause usability problems, including disorientation and the feeling of losing control. Adaptations are therefore often discussed in terms of costs and benefits for the users. However, design strategies to overcome the drawbacks of adaptations have received little attention in the literature. We have designed different adaptation patterns to increase the transparency and controllability of run time adaptations in our MyUI system. This paper presents an experimental user study to investigate the effectiveness and acceptability of the proposed patterns in different cost-benefit situations and for different users. The patterns turn out to increase the transparency and controllability of adaptations during the interaction. They help users to optimize the subjective utility of the system's adaptation behavior. Moreover, the results suggest that preference and acceptance of the different patterns depend on the cost-benefit condition.
\end{abstract}

Keywords: Adaptive user interfaces, design patterns, accessibility, user study, controllability.

\section{Introduction}

Self-learning and self-adaptive user interfaces offer big potential for accessible technology. In contrast to one universal design for all, personalized user interfaces can offer optimal levels of usability and accessibility for diverse users and context conditions by individual design solutions [1]. For personalization in accessible technologies there are two main approaches: adaptable and adaptive user interfaces (cf. [2]). Most adaptable user interfaces require complex configuration dialogues which can cause problems for the users [3] - especially for those who would benefit most from personalization. Therefore, adaptive user interfaces can be more effective in increasing accessibility. They include self-learning mechanisms to identify individual needs and system-initiated user interface adaptations to avoid and overcome barriers of use automatically and directly when they occur.

However, self-adaptive user interfaces also pose significant challenges in terms of usability, trust-worthiness and acceptability. The most prominent issues include confusing inconsistencies and the feeling of losing control over automatic changes in 
the user interface [4] [1]. Hence, mechanisms to improve the transparency and controllability of automatic adaptations will be a necessary feature of successful adaptive user interfaces. In this work, transparency means that users will be able to recognize and understand the automatic changes in the user interface and that confusion and disorientation will be avoided by making clear what has been changed and by supporting a "feeling of continuity between the user interface before and after adaptation" [5]. Moreover, it can be useful to explain the reasons for adaptations to the user in order to increase the predictability of the system behavior [4]. Adaptive user interfaces are said to be controllable if the user can control the intelligent and automatic adaptations. This can include opportunities for the user to prevent or actively accept adaptations, and to undo or override adaptations (cf. [6]). Many authors (e.g. [7] [8]) consider full user control over automatic adaptations as a major requirement of acceptable adaptive systems. For adaptive systems which aim at improving the accessibility, however, this claim might require reinterpretation. Impaired users sometimes need automatic adaptations in order to be able to access certain functionality and to control the interface at all. Trewin [9] argues that these users might experience an even increased rather than decreased level of overall control with automatic adaptations which require no extra interaction effort.

Adaptation mechanisms are error prone. Especially, incorrect adaptations challenge the transparency and controllability of an adaptive system. They lead to undesired user interface changes and make it difficult for the user to predict and understand the system behavior (cf. [10] [4]). Weld et al. [1] point out that incorrect adaptations are associated with costs for the user which have to be taken into account together with the potential advantages of an adaptive system. Systematic cost-benefit considerations cover also the costs of correct adaptations such as becoming aware of and leveraging the adaptation [11]. As a consequence, adaptive user interfaces will be successful only if the subjective benefits of the adaptations outweigh their costs [10]. Intelligent technologies will be limited in their ability to optimize individual cost-benefit ratios. Mechanisms to put the user into control of the system's adaptation behavior can be regarded as an effective means to optimize the subjective utility of automatic adaptations.

We recognize that the design of the adaptation process is a major issue for the usability and acceptability of adaptive user interfaces. In our MyUI system, we address this important field with dedicated Adaptation Patterns. They inform the users about intended or current adaptations. And they provide the users with dialogue mechanisms to optimize the costs and benefits of automatic adaptations by increasing their transparency and controllability [12]. This paper concentrates on an in-depth examination of the effectiveness and acceptability of the MyUI adaptation patterns from the users' perspective. This work makes the following contributions:

1. We introduce a systematic cost-benefit analysis of user interface adaptations for accessibility which serves as a basis for the experimental evaluation of adaptation dialogue mechanisms under different conditions.

2. The results of our study provide valuable insights into the interactions between the costs and benefits of an adaptation and the preferred level of user control. We discuss how the findings can be used for the design of effective adaptation dialogues. 


\section{Related Work}

The preconditions and problems of transparency in adaptive user interfaces are well described in the literature. Findlater and Gajos [10] report that adaptive menus are more understandable and predictable if they preserve spatial stability and if the adaptations are "elective", i.e. they can be ignored by the users. Tsandilas and Schraefel [13] point out the negative effect of poor adaptation accuracy on user performance in adaptive menus. They explain their findings by a decrease of user reliance on the system and thereby a decrease of subjective predictability of the system's behavior.

However, only few approaches to increase the transparency of automatic adaptations have been published. In a first systematic attempt, Dessart et al. [5] propose animated transitions for showing the adaptation process to the user. They develop a catalogue of "adaptation operations" (e.g. resizing, relocating, etc.) and suggest suitable animated transitions to support continuity in the perception of the dynamically changing user interface. Other approaches aim at a deeper user understanding of the system's adaptations by providing detailed explanations [14] or "by supporting the user in developing an adequate mental model of the systems' adaptation mechanisms" [15]. However, it seems questionable if and how these approaches can be applied to adaptations which try to compensate for perceptual, cognitive and motor impairments in users.

The problem of controllability has mainly been treated in terms of theoretic taxonomies rather than specific design guidelines. In the concept of PLASTIC USER INTERFACES, a "supra-UI" [16] or "meta-UI" [17] is envisaged by which the user can control the system's adaptations. Three levels of controllability are distinguished: At the lowest level, the user can only observe but not influence the adaptation process. At the intermediate level ("approbation"), the user can accept or reject an adaptation proposed by the system. The highest control level is characterized by a full user control over the adaptation ("specification") [16]. With Jameson's [4] suggestion to collect simple user feedback after performing an automatic adaptation (e.g., "I don't like what just happened"), the above mentioned approbation strategy can be further subdivided into a confirmation before and a confirmation after the adaptation.

Lavie and Meyer [18] compare four similar adaptation strategies with different levels of user control in a user study: a "manual" condition, in which the user performs all actions; "user selection" where the user selects one of the alternative adaptations suggested by the system; "user approval" where the user can accept or reject the system-initiated action; and "fully adaptive" where the system performs all actions and the user can just abort the adaptation process. The four strategies differ in their effectiveness in supporting a secondary task during a driving simulation session. In routine situations where the system can offer correct adaptations, the fully adaptive variant is the most beneficial for the users. In unfamiliar situations with inappropriate system adaptations, the other conditions with active user involvement perform better. This cost-benefit pattern is even more apparent in their second study with older participants. They benefit more from automatic adaptations when they are correct - but experience higher costs in cases of incorrect adaptations [18]. Even if Lavie and Meyer's experiment investigates adaptivity in the sense of automating the performance of a user task, 
their results can be easily transferred to adaptive user interfaces for improved accessibility. For users with more difficulties in using technology, the benefits of correct adaptations seem to clearly outweigh the costs of losing control over the system's adaptation behavior.

Another valuable contribution of Lavie and Meyer's work is that they analyze the utility of adaptations in combination with different control mechanisms. Earlier research has focused on the costs and benefits of different adaptations [19] [13] [9] [10]. But Lavie and Meyer [18] investigate how different adaptation control mechanisms can influence the costs and benefits of the same adaptations.

However, for a systematic analysis of the costs and benefits of adaptations and their interactions with adaptation control mechanisms, their study has two main shortcomings. Firstly, the specific influence of costs and benefits cannot be separated since only two of the four possible cost-benefit conditions (one with high benefit and one with high cost) have been compared. Secondly, only objective performance measures have been taken. However, the predominant disadvantages of adaptive systems as reported in the literature do not relate to performance but to more subjective concepts such as experienced transparency and the feeling of control.

\section{Adaptations in the MyUI System}

\subsection{Overview of the MyUI System}

This section summarizes the main features of the MyUI system (see [12] for a more detailed description) to provide the conceptual background for the experimental user study. MyUI aims at mainstreaming accessible and highly individualised ICT products. Instead of developing dedicated user interfaces for specific user groups with certain disabilities, MyUI tackles the problem by providing a generic infrastructure to enhance mainstream products with automatically generated user interfaces which adapt to diverse user needs, different devices, and changing context conditions. MyUI adaptations include changes in the presentation formats and modalities, interaction mechanisms and navigation paths. MyUI takes a modular approach to adaptive user interfaces relying on the composition of multimodal user interface design patterns. Individual accessibility is achieved by combining patterns which provide proven solutions for specific interaction situations and characteristics of the user, environment and device [12].

MyUI strives for a smooth and natural adaptation process by avoiding the need of an initial configuration or user enrolment process. The MyUI system senses and interprets information about the user and the context of use directly during the interaction and refines the profiles accordingly. Profile changes immediately lead to automatic run-time adaptations of the user interface. This closed loop of self-learning user and context profiles and system-initiated run-time adaptations supports a continuously improved fit between the adaptive user interface and individual user needs. Thus, also altering user capabilities as typical in the course of aging and rehabilitation (cf. [20]) can be covered. Moreover, barriers of use can be overcome directly when they occur. 
Profile changes that lead to user interface adaptations are triggered by a number of sensor events. Most triggering events are detected from the user's interaction behaviour with the current user interface. Examples include time-outs (the user does not react to a system prompt within a certain time frame), repeated undos (the user seems to repeatedly select wrong options by accident) and detours. Moreover, also hardware-based sensors as, for example, eye tracking in order to capture the user's attention can be used in the MyUI user profiling process (cf. [21]). In order to avoid too many changes on the user interface and to compensate for the uncertainty of the sensor-based recognition procedures, user profiling involves statistical processing mechanisms. As a result, not every single sensor event will immediately trigger an adaptation, but adaptations will be triggered only if a certain threshold in the user profile is exceeded. This supports user interface stability. But on the other hand, transparency suffers. Users can have problems in understanding and anticipating automatic adaptations. It can be difficult to establish a clear connection between the adaptations and their causes, i.e. the triggering events.

\subsection{MyUI Adaptation Patterns}

The MyUI design patterns repository [22] includes different types of patterns which serve distinct functions in the adaptation process. There are patterns for creating and updating a user interface profile which includes global variables to define general settings throughout the entire user interface (e.g. font size). Other patterns provide alternative user interface components and elements for current interaction situations. Finally, adaptation patterns describe the transition from one instance of the user interface to another. Their main goal is to assure high levels of transparency and controllability for the user during automatic adaptations. There are two types of adaptation patterns:

- Adaptation rendering patterns specify the transitions from the user interface before the adaptation to the user interface after the adaptation. They use animations as recommended by [5] to create continuity in the course of adaptations. The animated transitions draw the user's attention to the changing screen areas and make clear the relationship between the new, adapted user interface and the former user interface. Examples include animations to grow small fonts to bigger fonts or to move elements from one area to another. Adaptation rendering patterns are always part of an adaptation process as specified by the adaptation dialogue patterns.

- Adaptation dialogue patterns specify the dialogue around an adaptation to make sure that the user is aware of the adaptation and can control the system's adaptation behaviour. Typical elements of an adaptation dialogue include a notification and interaction options for the user to control the system's adaptation behaviour. The MyUI patterns repository includes different adaptation dialogue patterns with different levels of user control.

The main objective of this paper is to investigate the effectiveness and acceptability of the different adaptation dialogue patterns in different cost-benefit situations and to understand their contribution to optimize the subjective costs and benefits of an 
adaptation as experienced by the user. Before presenting the experimental design and the results of our user study, we describe the patterns and their assumed effects on the usability and acceptability of automatic run-time adaptations.

\subsection{Adaptation Dialogue Patterns}

Automatic Adaptation without Adaptation Dialogue (baseline). This pattern can be regarded as a baseline condition. It is currently not used in the MyUI system. The adaptation is triggered and performed automatically. Besides the animated transitions of the involved adaptation rendering patterns, there is no additional indication of the current adaptation. The user has no opportunity to control, cancel or undo the adaptation.

Automatic Adaptation with Implicit Confirmation (AI). During the automatically triggered adaptation, an animated icon notifies the user about the on-going adaptation in a dedicated adaptation area on the screen. When the adaptation is finished the end user can undo the adaptation via a button in the adaptation area. Moreover, the adaptation area offers a permanent access to the user interface profile and the user profile.

Compared to the baseline condition, this pattern is expected to slightly increase the transparency and controllability by adding the animated icon and the undo opportunity. However, both mechanisms are unobtrusive and require a certain level of system experience and attention. On the other hand, this type of adaptation control will not decrease the interaction efficiency as no additional steps are required if the user agrees with the proposed adaptation.

Explicit Confirmation before Adaptation (EB). Before the user interface is adapted, a dialogue box is displayed which requests the user to explicitly accept or reject the adaptation. A preview of the user interface after the adaptation supports the user's decision. If the user rejects the adaptation, the dialogue box is closed and the current user interface is not changed. If the user accepts the adaptation or if the system receives no user input (time-out), the dialogue box is closed and the adaptation is carried out.

This pattern is assumed to strongly increase the transparency and controllability. The dialogue box offers an obvious hint to the planned adaptation and does not start the adaptation before the user provides an explicit agreement. However, it interrupts the current interaction and requires an extra step in the dialogue. This might cause problems for some users and reduce the efficiency - especially with adaptations which are perceived beneficial by the user.

Explicit Confirmation after Adaptation (EA). When the automatically triggered adaptation has been finished, a dialogue box asks the user if the changes shall be kept or undone. In case of rejection, the adaptation is recalled. In case of acceptance or a time-out event, the adaptation is kept.

This pattern is similar to the EB pattern. We expect that due to the delayed confirmation dialogue, the transparency and controllability gains will not be as high as with the EB pattern. It also requires the described extra step. 


\section{$4 \quad$ Experimental User Study}

\subsection{Goals}

Our study had two main goals. First, we wanted to validate the MyUI adaptation dialogue patterns and evaluate their effectiveness and acceptability compared to the baseline condition. Second, we wanted to gain insights in how to implement the patterns in the MyUI adaptation engine. Especially, we wanted to know if we can use a simple adaptation process with only one design pattern which is preferable under all conditions or if more complex adaptation process behaviour will be required in order to present different patterns in different cost-benefit conditions and to different users.

\subsection{Hypotheses}

User interface adaptations can support the accessibility by detecting and overcoming specific problems or barriers of use during the interaction. In these cases, adaptations will provide a benefit for the user. However, wrong system assumptions about individual user requirements can lead to adaptations which are not beneficial for the user. On the other hand, adaptations can also cause costs by raising the effort for task completion. This leads us to the distinction of adaptations with low vs. high costs and benefits:

- Adaptations with high benefits help to overcome an individual interaction barrier, e.g. increase the font size when the user has vision problems.

- Adaptations with low benefits have no positive usability effect for the user in the current situation, i.e. the user does not experience a barrier of use before the adaptation occurs. This condition represents useless adaptations as an effect of user profiling errors.

- Adaptations with high costs require an extra interaction effort compared to the user interface before adaptation, e.g. after increasing the font size, the user needs to scroll down the page in order to access the desired information.

- Adaptations with low costs do not require extra interaction effort.

The four cost-benefit conditions which result from a $2 \times 2$ combination of low and high costs and benefits are systematically produced in our experiment and serve as the experimental context conditions for testing the above described adaptation dialogue patterns. For each pair of pattern and cost-benefit condition, subjective user assessments are recorded to evaluate the perceived transparency, controllability, comfort of use and acceptance of the patterns as well as the subjective costs and benefits of the adaptations.

The following four research hypotheses are investigated:

1. The adaptation patterns AI, EB and EA perform better than the baseline in terms of subjective transparency, controllability, as well as acceptability and user preference.

2. The adaptation patterns differ in their capability to support users in optimizing the subjective utility of an adaptation. We assume that control mechanisms can help users to minimize the subjective costs of undesired adaptations. 
3. In different cost-benefit conditions, users prefer different adaptation patterns and the patterns receive different levels of user acceptance. We assume that high-cost adaptations are associated with a stronger wish for control while high-benefit adaptations will lead to preferring more efficient patterns.

4. User preference and acceptance of the patterns differ for different user groups, e.g. users with higher levels of technology anxiety prefer patterns with more control.

\subsection{Methods}

Participants. For this experiment, twelve older adults were recruited. They were paid $€ 50$ to participate for one test session of about 90 minutes. The participants were between the ages of 49 and 73 , with an average of 63 years; there were seven females and five males. None of them reported significant accessibility problems. Three had minor reading problems with small fonts, two said they preferred bigger keys on remote controls, and four reported problems with the understandability of instructions or technical terms in interactive products. Six described their ICT literacy level as medium, three as low, and three as fairly good.

The selection of able-bodies participants for this experiment was deliberate. In accordance with ISO 9241-171 [23], accessibility does not only apply to disabled users but also to users with temporary impairments or users who experience difficulties in particular situations, etc. Common to all users who benefit from an adaptive and accessible system like MyUI is the experience of a barrier which is then removed by an adaptation - regardless of the many different possible reasons and disabilities. All our users were at ages where barriers of use can arise quite suddenly, even without disabilities. Involving only disabled users might cause a bias as these users might have found their ways to cope with accessibility issues and they might be prejudiced against intelligent solutions from prior experiences. Moreover, our able-bodied sample helped us to exclude undesired side effects of disabilities which are not covered by the design solutions presented in the experiment. Working with participants without disabilities allows us to fully control the cost-benefit conditions.

Thus, a major assumption of our study is that preference and acceptance of the adaptation patterns will not depend on a specific type or level of impairment but mainly on the experienced costs and benefits of the offered adaptation.

Apparatus. The experiment was conducted in a typical usability lab setting. Three Windows-based computers were used. The first computer ran the interactive test prototype which simulated an adaptive interactive TV system with a main menu, and an email application to receive, view, compose and send emails. This computer was connected to a 32" PHILIPS TV screen (32PFL7605H 12) and was controlled by an infrared remote control as commonly used with TV devices. The other two computers were used for accessing and manipulating the MyUI user and context management infrastructure to start adaptations by changing user profile variables in a Wizard-of-Oz manner. The participants were sitting on a sofa in front of the TV screen with a viewing distance of about 2 meters. They had to work on different tasks with the prototype system while using the remote control. 
Tasks, Adaptations and Cost-Benefit Conditions. The following three test tasks were used. During each task, a system-initiated adaptation was performed:

1. Task: Select an application from the iTV main menu.

Adaptation: Add numeric key labels to the menu items in order to allow for direct selection via numeric keys instead of cursor navigation. This adaptation is expected to help motor impaired users with difficulties in precise and repeated key presses.

2. Task: Select an email from the email inbox.

Adaptation: Replace sender names by photos. This adaptation shall help cognitively impaired user who have difficulties in language reception or text reading.

3. Task: Read a received email message.

Adaptation: Increase font size. This adaptation helps users with low vision.

With these pairs of tasks and adaptations, the four cost-benefit conditions were implemented. While the costs could be easily manipulated by the task instructions and the user interface design, the benefit conditions required special attention. The older participants were deliberately selected to have no considerable accessibility problems, i.e. none of the adaptations above would usually be beneficial for them. In order to systematically vary the benefit level we artificially induced interaction barriers (figure 1) which could be removed by user interface adaptations. In the first task (menu
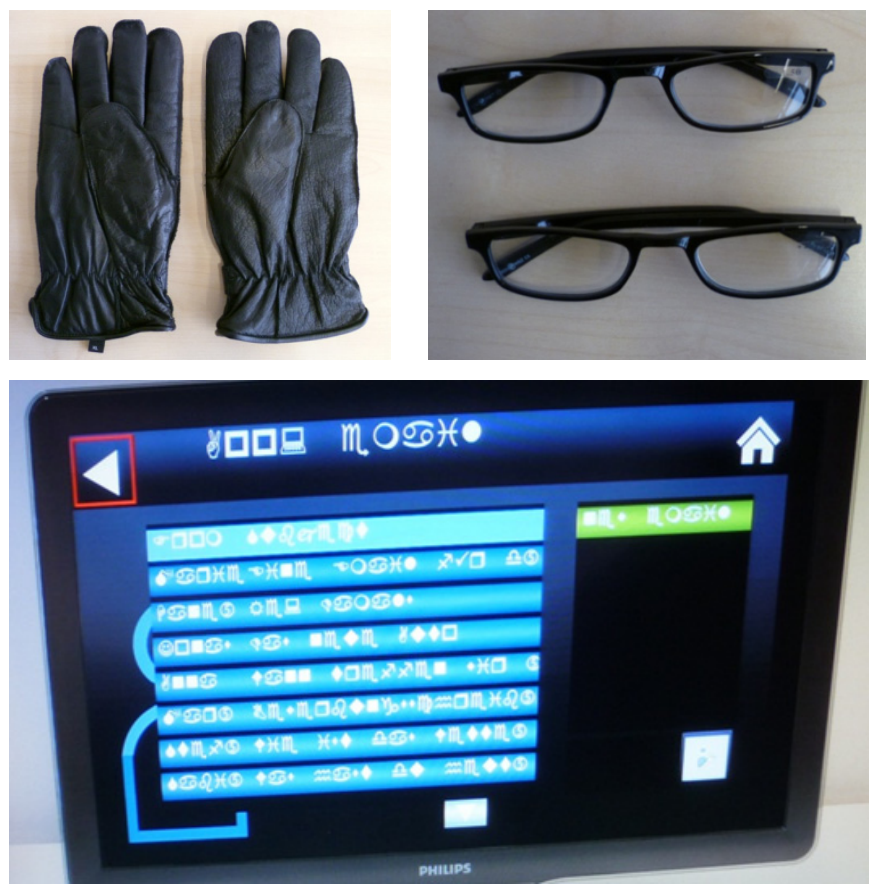

Fig. 1. Gloves, glasses and nonsense font (Wingdings) to systematically vary the benefits of user interface adaptations 
selection), the high-benefit condition was created by instructing the users to wear leather gloves in size XL which should pose a significant barrier to key pressing on the remote control. As a beneficial adaptation in this condition, enabling direct numeric key selection reduced the required number of key presses. In a similar manner, the Windows font type "Wingdings" was used for all text elements in the second task (inbox) so that the text output did not make any sense for the participants. In the high-benefit condition, this artificial barrier was overcome by adding sender photos to the email items so that the participants could easily recognize the searched email. In the third task (read email), glasses with dioptre values of +1.5 and +2.5 were used to pose reading problems which could be alleviated by increasing the font size adaptations in the high-benefit condition.

For the example of the task "read email", figure 2 shows how the four cost-benefit conditions were produced by different instructions and artificial barriers of use.

It is important to explain that the artificial barriers (gloves, nonsense font type and glasses) were not intended to simulate any motor, cognitive or perceptual impairment in a realistic way. It would not be reasonable to assume that wearing gloves, for example, could create an interaction experience which is similar to the experience of a user with impaired finger precision. Moreover, we do not claim that this approach could cover the entire area of potential accessibility problems in a representative manner. However, it was our goal to systematically vary the benefits of automatic adaptations for accessibility. Therefore, it was important to produce the general underlying mechanisms of beneficial adaptations: The user experiences an interaction barrier and is not able to complete a given task. Then a system-initiated adaptation occurs and helps the user to overcome the problem. In order to prove the validity of

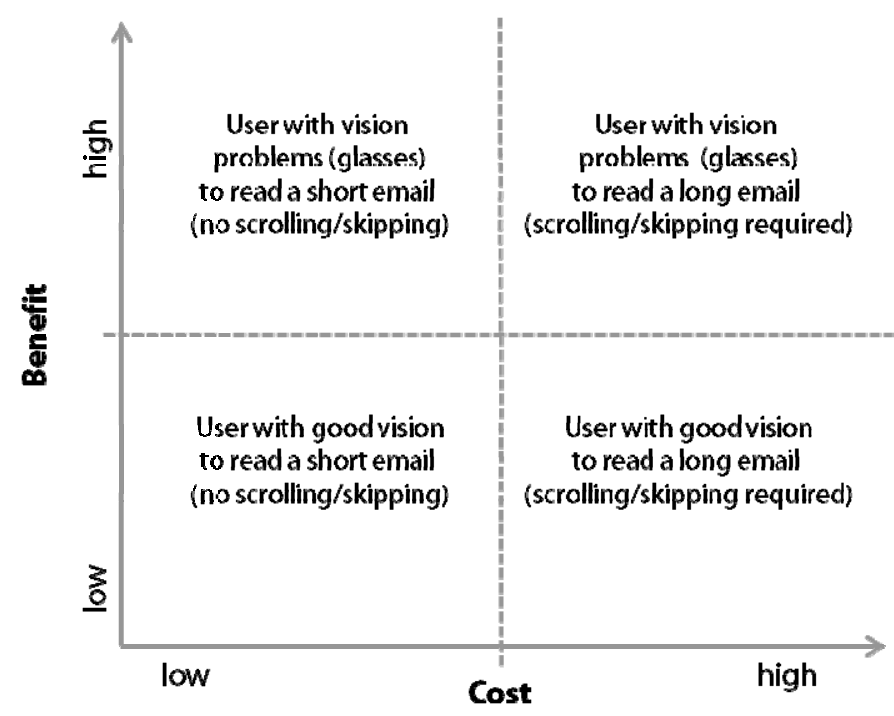

Fig. 2. Cost-benefit conditions for the adaptation of increasing the font size during the read email task (example) 
our approach, we asked the participants to rate the subjective benefit of each experienced adaptation. We yielded a substantial level of agreement between the intended benefit condition and the subjective benefit ratings (Cohen's Kappa $\kappa=0.729, n=96$ ). The experimental variation of the costs of adaptations is supported by a fair agreement of experimental conditions and user ratings (Cohen's Kappa $\kappa=0.375$, n=96).

Experimental Design and Measures. The study was a 4 (cost-benefit condition) x 4 (adaptation dialogue patterns) within-subjects design. Task and adaptation type was not used as another independent variable but was varied systematically to make sure that our findings were not restricted to only one type of task and adaptation and that they could be generalized. Each of the three task/adaptation conditions was performed twice in all $4 \times 4$ test cases. The total of 96 test conditions was randomly assigned to the twelve participants so that each participant performed eight tasks. And each participant experienced two of the three task/adaptation conditions for four times, each with all four cost-benefit conditions and all four adaptation dialogue patterns - the first task/adaptation condition naïve and the second set of four runs after they were introduced into the different adaptation patterns. The restriction to two of the three task types was due to time limitations. In a pre-study we found that eight tasks were the possible maximum within the intended time frame of 90 minutes per session.

The dependent variables of the study included subjective assessments of the adaptation patterns after each of the eight test cases per participant on a four-point Likert scale for:

- Transparency (two items: "The design is good in communicating that a change is being processed" and "The design is good in communicating what the user can do in order to stop or undo the change")

- Controllability (two items: "I feel in full control of the system, its appearance and changes" and "It is easy to control the system and its appearance and changes.")

- Comfort of use (two items: "The design is good in supporting a comfortable system use" and "The design is good in avoiding unnecessary user input or interaction steps.")

- General acceptance (one item: "All in all, I like the presented style of dialogue when the system is adapting to my individual needs.")

Moreover, the participants assessed the costs and benefits of the eight experienced adaptations on four-point Likert scales. Finally, the participants indicated their preferred pattern for each of the four latter test cases and a general preference in the post-task interview at the end of the session.

Procedure. The overall procedure of a single test session was as follows:

1. After a welcome and introduction to the test situation, a questionnaire was used to obtain information on the user's ICT literacy, technology interest and technology anxiety.

2. The participants had to complete a sequence of four interaction tasks with the interactive TV application. During each interaction, an adaptation occurred with a different hypothesized cost-benefit level and a different adaptation dialogue 
pattern. In the high-benefit condition, the adaptation was triggered (Wizard of Oz) after a time-out of 10 seconds when the participant was stuck with the artificial barrier. In the low benefit condition, the adaptation was triggered 20 seconds after the participant has started to work on the task with no obvious triggering event. After each task the participants assessed the transparency, controllability, comfort of use and overall acceptance of the adaptation pattern and the subjective costs and benefits of the adaptation via a questionnaire.

3. After the first four tasks, the participants were interviewed about the experienced adaptation patterns. If they did not recognize the differences, the test facilitator explained the different adaptation dialogue patterns.

4. The participants worked on another sequence of four interaction tasks with different intended cost-benefit conditions and different adaptation patterns. Again the questionnaire for the subjective assessment was filled after each task and the participants were asked to indicate which of the four different patterns they would prefer in the current situation.

5. A final interview was conducted to discuss general preferences regarding the pat-terns and to collect qualitative user feedback.

\subsection{Results}

Transparency, Controllability, Comfort of Use and Acceptance. The adaptation dialogue patterns AI, EB and EA were rated significantly better than the baseline adaptation in terms of transparency, controllability and acceptability (figure 3). Data analysis referred to the number of positive assessments of a criterion. For a two-item

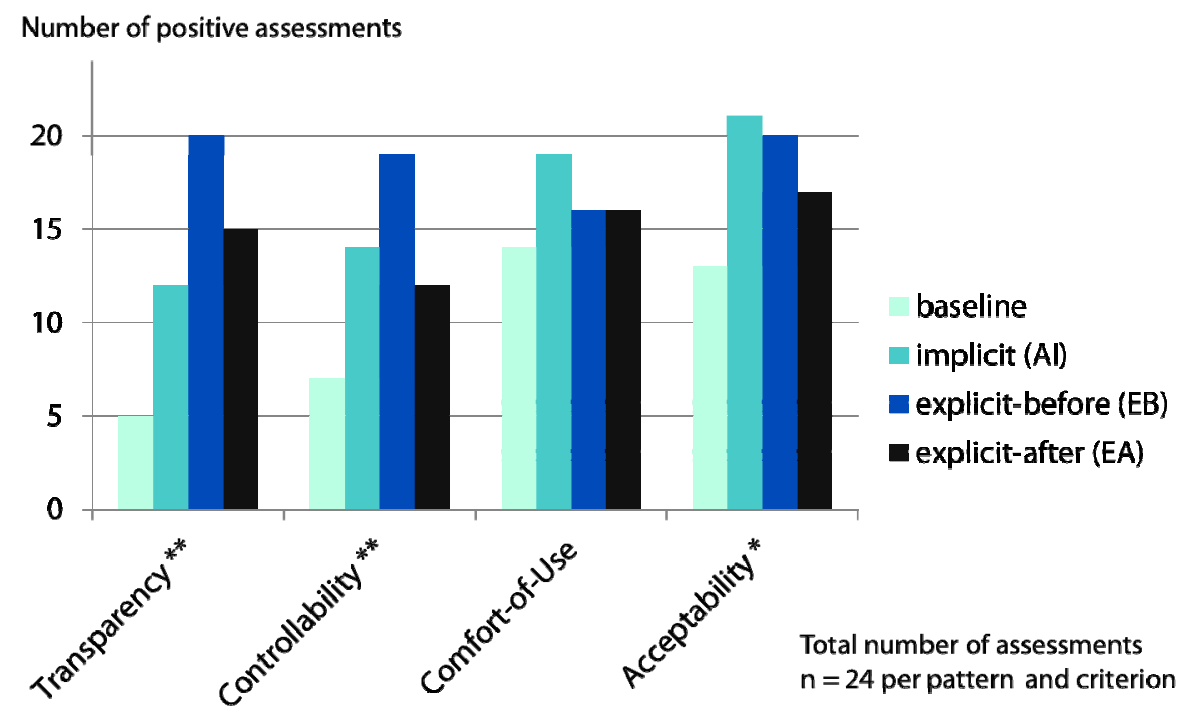

Fig. 3. Numbers of positive user assessments of transparency, controllability, comfort-of-use and acceptability for the different adaptation dialogue patterns 
concept, this requires both items assessed positive (3 or 4 on the four-point Likert scale). A chi-square test against the $\mathrm{HO}$ of equally distributed user ratings for all four patterns revealed significant differences for transparency $\left(\chi^{2}(3, \mathrm{~N}=96)=19.80\right.$, $\mathrm{p}<0.001 * *)$, controllability $\left(\chi^{2}(3, \mathrm{~N}=96)=12.42, \mathrm{p}=0.006^{* *}\right)$ and acceptability $\left(\chi^{2}(3\right.$, $\left.\mathrm{N}=96)=8.38, \mathrm{p}=0.039^{*}\right)$. As assumed, the dialogue pattern EB yielded the best assessments for transparency and controllability. The comfort-of-use ratings did not differ between the four patterns.

The analysis of the user preferences yielded similar results (table 1). The patterns differed significantly in their user preference for the single test tasks $\left(\chi^{2}(3, \mathrm{~N}=48)=\right.$ $11.00, \mathrm{p}=0.012 *)$. In only 3 out of 48 test cases $(6.25 \%)$, the baseline condition was the preferred adaptation mechanism. The patterns AI and EB were preferred by most users.

Table 1. Preferred adaptation dialogue patterns (absolute frequencies)

\begin{tabular}{lccll}
\hline & baseline & AI & EB & EA \\
\hline Preference per test case $(\mathrm{n}=48) *$ & 3 & 17 & 17 & 11 \\
General preference $(\mathrm{n}=12)$ & 1 & 5 & 5 & 1 \\
\hline
\end{tabular}

Control of Subjective Costs of Adaptations. Considering all 96 test cases, the adaptation dialogue patterns did not differ in their capability to reduce the subjective costs $\left(\right.$ Cost $\left._{\text {subj. }}\right)$ compared to the costs as intended by the experimental condition $\left(\right.$ Cost $\left._{\text {int. }}\right)$. The number of cases where participants reported low subjective costs while being in a high-cost test condition, were equal for all four patterns $\left(\chi^{2}(3, \mathrm{~N}=96)=\right.$ $0.54, \mathrm{p}=0.91)$. However, when users were aware of the different adaptation patterns after the review, significant differences were found $\left(\chi^{2}(3, \mathrm{~N}=48)=8.76, \mathrm{p}=0.033^{*}\right)$. According to our assumptions, the two patterns with explicit confirmations seemed to be most effective in reducing the costs of high-cost adaptations (table 2).

For the subjective benefit ratings, no effects of the patterns were found. It seems that even patterns which require extra effort do not reduce the advantages of adaptations which are beneficial for the users.

Table 2. Adaptation dialogue patterns and their capability to control the costs of adaptations. The table shows absolute frequencies of cases where participants report low subjective costs while being in a high-cost test condition ("Cost $t_{\text {subj. }}<$ Cost $_{\text {int. }}$ ").

\begin{tabular}{lllll}
\hline & baseline & AI & EB & EA \\
\hline All test cases $(\mathrm{n}=96)$ & & & & \\
Cost $_{\text {subj. }}<$ Cost $_{\text {int. }}$ & 6 & 7 & 8 & 8 \\
Cost $_{\text {subj. }} \geq$ Cost $_{\text {int. }}$ & 18 & 17 & 16 & 16 \\
\hline After review $^{1}(\mathrm{n}=48) *$ & 1 & 1 & 5 & 6 \\
Cost $_{\text {subj. }}<$ Cost $_{\text {int. }}$ & 11 & 11 & 7 & 6 \\
Cost $_{\text {subj. }} \geq$ Cost $_{\text {int. }}$ & 11 & & \\
\hline
\end{tabular}

1 "After review": the users are aware of the different patterns 
Preference and Acceptance Depend on Cost-Benefit Conditions. In contrast to our assumption, user preferences did not differ between different cost conditions. A reason might be that already in the low-cost conditions, the patterns with high control levels were preferred very often. A further effect into the expected direction might have been made more difficult by a ceiling effect.

However, a significant interdependency of the benefits as intended by the experimental conditions and the pattern preferences was discovered $\left(\chi^{2}(3, \mathrm{~N}=48)=8.07\right.$, $\mathrm{p}=0.045^{*}$ ) (table 3). Especially, the implicit confirmation pattern AI was more often preferred with highly beneficial adaptations. This effect was even stronger for the subjective benefit as reported by the users $\left(\chi^{2}=15.70, \mathrm{df}=3, \mathrm{p}=0.001^{* *}\right)$. As predicted, when the benefits of an adaptation were high enough, controllability loses its importance and the more efficient implicit confirmation (AI) was preferred.

The different cost and benefit conditions had no significant effect on the acceptance ratings of the different patterns, when regarding the intended utility. However, subjective costs and acceptance judgments were significantly associated $(\chi 2(7, N=96)$ $=22.21, \mathrm{p}=0.002 * *)$. Especially, the baseline pattern and EA received less positive acceptance judgments when the adaptation caused higher subjective costs. This result indicates that adaptation mechanisms with insufficient user control will be disliked when adaptations with high subjective costs occur. On the other hand, acceptance judgments were also significantly associated with the subjective benefits of the adaptation $\left(\chi 2(7, \mathrm{~N}=96)=14.38, \mathrm{p}=0.045^{*}\right)$. Especially, the baseline pattern gained higher acceptance with high-benefit adaptations. This finding is in line with the above reported result of less important control mechanisms for beneficial adaptations.

Table 3. Absolute frequencies of user preferences for the different adaptation dialogue patterns under the experimental conditions of low vs. high intended costs and benefits

\begin{tabular}{lllll}
\hline & baseline & AI & EB & EA \\
\hline Intended costs & & & & \\
Low $(n=24)$ & 2 & 7 & 8 & 7 \\
High $(n=24)$ & 1 & 10 & 9 & 4 \\
\hline Intended benefits * & 1 & 5 & 13 & 5 \\
Low $(n=24)$ & 2 & 12 & 4 & 6 \\
High $(n=24)$ & & & & \\
\hline
\end{tabular}

Influence of User Characteristics. The collected measures of individual ICT literacy, technology interest and technology anxiety were not associated with the users' acceptance and assessment of the patterns, except for the comfort-of-use judgements were significant interdependencies with ICT literacy $\left(\chi^{2}(7, \mathrm{~N}=96)=24.73\right.$, $\left.\mathrm{p}<0.001^{* *}\right)$ and technology interest $\left(\chi^{2}(7, \mathrm{~N}=96)=15.02, \mathrm{p}=0.036^{*}\right)$ were found. Users with higher levels of ICT literacy rated the comfort of use of EB better whereas users with low ICT literacy rated the comfort of use of EA better. Finally, users with 
low technology interest judge the comfort of use of EA better than users who are more interested in technology.

\section{Discussion}

Our work provides several interesting insights with respect to control mechanisms for automatic user interface adaptations. Some of our initial assumptions could not be confirmed by the empirical results. The rigid nonparametric statistical analyses might have been responsible for a relatively low statistical power. Our main findings can be summarized as follows:

The two adaptation dialogue patterns AI and EB have clearly shown to be best in terms of transparency and controllability. It seems that an optimal adaptive system will need both approaches: a quick and efficient adaptation process which provides only an undo for unsuccessful adaptations and a more obvious and explicit dialogue which waits for a user confirmation before changes are executed. The two preferred patterns are quite different and have their specific advantages under different conditions.

The explicit confirmation pattern proved to be more appropriate for situations in which adaptations are likely to cause costs for the user. To a certain degree, it seems that explicit control mechanism can reduce the negative impressions of automatic adaptations. In our experiment, the users still had to face the same inconveniences. But the fact that they can decide about the system's behaviour seems to alleviate the subjective disadvantages. On the other hand, the extra interaction step needed for the explicit conformation did not reduce the advantages of a helpful adaptation.

However, in situations where adaptations provide great benefits to the user, the implicit adaptation dialogue (AI) was clearly preferred. In this regard, our experiment confirms Trewin's [9] claim of a minor role for user control in adaptive user interfaces for accessibility. Even our baseline pattern with poor overall ratings for transparency and controllability was significantly better accepted when the users experienced a beneficial adaptation.

Our analysis of certain user characteristics and their influence on the subjective assessments of the different patterns brought the EA pattern into the discussion again. The three tested characteristics ICT literacy, technology interest and technology anxiety are covered by the MyUI user profile. It would be very easy to present the EA pattern to users with low levels of ICT literacy and technology interest. But the re-ported interaction effect is restricted to the EA pattern. And its absolute rating levels are - even for the most positive cases - still not better than for the other two adaptation dialogue patterns AI and EB.

Therefore, a combination of the two preferred adaptation dialogue patterns seems most appropriate. Based on these findings, we will refine the MyUI adaptation engine in a way that adaptations which cause only minor changes (i.e. changes without switching to other interaction design patterns, cf. [12]) will be associated with the implicit design pattern AI. Other adaptations which cause more substantial changes, 
e.g. substituting one user interface component by another, will engage the explicit adaptation dialogue pattern EB.

We have implemented a novel experimentation approach which aimed at producing generalizable results. Our cost-benefit classification aimed at generalizing from specific user interface adjustments and our procedure of systematically varying the adaptations' costs and benefits seemed to be effective in generalizing from specific impairments. If our assumptions are valid, we can generalize the findings to the design of control mechanisms for adaptive systems in general.

However, if the preference of control mechanisms depended more on specific levels and types of impairments rather than the experienced costs and benefits of an adaptation, a study with actually impaired user would be indispensable. Another limitation of our study concerns the fact that we have explained the different adaptations to the users after the first half of the experiment. This might have biased attention and awareness for the adaptation process. It is not clear how the findings apply to the real world where some users might never pay attention to the way adaptations are carried out. Finally, we were mainly interested in the preconditions of user acceptance for adaptive user interfaces. Therefore, we concentrated on aspects of the user experience and subjective ratings. In order to gain a wider view on the topic, it might be interesting to also collect objective data, e.g., about improvements in task performance for the different adaptation patterns.

In order to further validate this work we suggest a similar study with a less controlled but more realistic setting. This includes involving users with actual accessibility issues and presenting adaptations which specifically fit their individual needs. This future study shall also provide the opportunity to investigate if similar results can be achieved when the users experience the adaptation behaviour over a longer period of time without getting explicit explanations about the adaptation patterns.

\section{Conclusion}

The MyUI adaptation dialogue patterns mark a valuable approach to improve the transparency and controllability of automatic run-time adaptations for increased accessibility. We have presented a controlled lab experiment to validate their effectiveness and acceptance in different cost-benefit conditions. The systematic variation of cost-benefit conditions allowed us to investigate the manifold interactions between the utility of an adaptation and possible mechanisms to put the user in control of system-initiated adaptations.

Our study revealed strong support for two of the proposed adaptation patterns: "Automatic adaptation with implicit confirmation" (AI) and "Explicit confirmation before adaptation" (EB). On the basis of their specific advantages in different cost-benefit situations, we conclude to use both patterns in our MyUI adaptation frame-work. And we advise other designers to use corresponding confirmation strategies in their adaptive systems. AI is best for adaptations with great advantages for the user. It provides an unobtrusive notification of the current adaptation and offers the opportunity to undo the adaptation. In situations where high costs for the user are 
expected, EB should be used. This pattern has proven to reduce the subjective costs of an adaptation and offers very clear control mechanisms by requesting an explicit user confirmation before the adaptation is executed.

Acknowledgments. The research leading to these results has received funding from the European Union's Seventh Framework Programme under grant FP7-ICT-248606. The authors acknowledge the help of other partners in the MyUI consortium in this work.

\section{References}

1. Weld, D., Anderson, C., Domingos, P., Etzioni, O., Lau, T., Gajos, K., Wolfman, S.: Automatically personalizing user interfaces. In: Proceedings IJCAI 2003, pp. 1613-1619. Morgan Kaufmann, San Francisco (2003)

2. Findlater, L., McGrenere, J.: A comparison of static, adaptive, and adaptable menus. In: Proceedings CHI 2004, pp. 89-96. ACM, New York (2004)

3. Mackay, W.E.: Triggers and barriers to customizing software. In: Proceedings CHI 1991, pp. 153-160. ACM, New York (1991)

4. Jameson, A.: Understanding and Dealing with Usability Side Effects of Intelligent Processing. AI Magazine 30(4), 23-40 (2009)

5. Dessart, C.-E., Motti, V.G., Vanderdonckt, J.: Showing user interface adaptivity by animated transitions. In: Proceedings EICS 2011, pp. 95-104. ACM, New York (2011)

6. Wobbrock, J.O., Kane, S.K., Gajos, K.Z., Harada, S., Froehlich, J.: Ability-based design: Concept, principles and examples. ACM Transactions on Accessible Computing 3(3), 1-27 (2011)

7. Norman, D.: How might people interact with agents. Communications of the ACM 37(7), 68-71 (1994)

8. Vanderdonckt, J., Coutaz, J., Calvary, G., Stanciulescu, A.: Multimodality for Plastic User Interfaces: Models, Methods, and Principles. In: Tzovaras, D. (ed.) Multimodal user Interfaces: Signals and Communication Technology, pp. 61-84. Springer, Berlin (2008)

9. Trewin, S.: Automating accessibility: the dynamic keyboard. ACM SIGACCESS Accessibility and Computing (77-78), 71-78 (2003)

10. Findlater, L., Gajos, K.Z.: Design Space and Evaluation Challenges of Adaptive Graphical User Interfaces. AI Magazine 30(4), 68-73 (2009)

11. Gajos, K.Z., Czerwinski, M., Tan, D.S., Weld, D.S.: Exploring the design space for adaptive graphical user interfaces. In: Proceedings AVI 2006, pp. 201-208. ACM, New York (2006)

12. Peissner, M., Häbe, H., Janssen, D., Sellner, T.: MyUI: Generating accessible user interfaces from multimodal design patterns. In: Proceedings EICS 2012, pp. 81-90. ACM, New York (2012)

13. Tsandilas, T., Schraefel, M.C.: An Empirical Assessment of Adaptation Techniques. In: CHI 2005 Extended Abstracts, pp. 2009-2012. ACM, New York (2005)

14. Bunt, A., Conati, C., McGrenere, J.: A Mixed-Initiative Approach to Interface Personalization. AI Magazine 30(4), 58-64 (2009)

15. Kühme, T.: A user-centered approach to adaptive interfaces. In: Gray, W.D., Hefley, W.E., Murray, D. (eds.) Proceedings IUI 1993, pp. 243-245. ACM, New York (1993) 
16. Calvary, G., Serna, A., Coutaz, J., Scapin, D., Pontico, F., Winckler, M.: Envisioning Advanced User Interfaces for e-Government Applications: a Case Study. In: Assar, S., Boughzala, I., Boydens, I. (eds.) Practical Studies in E-Government: Best Practices from Around the World, pp. 205-228. Springer (2011)

17. Coutaz, J.: User interface plasticity: model driven engineering to the limit! In: Proceedings EICS 2010, pp. 1-8. ACM, New York (2010)

18. Lavie, T., Meyer, J.: Benefits and costs of adaptive user interfaces. Int. J. Hum.-Comput. Stud. 68(8), 508-524 (2010)

19. Horvitz, E.: Principles of mixed-initiative user interfaces. In: Proceedings CHI 1999, pp. 159-166. ACM, New York (1999)

20. Sloan, D., Atkinson, M.T., Machin, C., Li, Y.: The potential of adaptive interfaces as an accessibility aid for older web users. In: Proceedings W4A 2010, Article 35, 10 pages. ACM, New York (2010)

21. Hernández, J.A., Larrabeiti, D., Strnad, O., Schmidt, A.: Prototype for user context management infrastructure and user modelling. Public deliverable of the MyUI project (2011), http://www.myui.eu/docs/MyUI_D1-2_final.pdf

22. MyUI Design Patterns Repository, http: / / myuipatterns. clevercherry.com

23. ISO (International Organization for Standardization): ISO 9241-171:2008 Ergonomics of human-system interaction - Part 171: Guidance on software accessibility (2008) 